\title{
Use of bedaquiline and delamanid in diabetes patients: clinical and pharmacological considerations
}

This article was published in the following Dove Press journal:

Drug Design, Development and Therapy

7 December 2016

Number of times this article has been viewed

Minhui $\mathrm{Hu}^{\prime}$

Chunlan Zheng'

Feng $\mathrm{Gao}^{2}$

'Department of Internal

Medicine - Section 5, Wuhan

Pulmonary Hospital (Wuhan

Tuberculosis Control Institute),

${ }^{2}$ Department of Endocrinology,

Union Hospital, Tongji Medical

College, Huazhong University

of Science and Technology, Wuhan,

People's Republic of China
Correspondence: Feng Gao

Department of Endocrinology, Union

Hospital, Tongji Medical College,

Huazhong University of Science and

Technology, 1277 jiefang Avenue, Wuhan

430022, People's Republic of China

$\mathrm{Tel}+8613971525208$

Email gaofengwh@aliyun.com

\begin{abstract}
Antituberculosis (anti-TB) treatment may be affected by both diabetes and hypoglycemic agents in patients with these 2 comorbidities. However, data supporting this conclusion relate only to standard anti-TB therapies. Sirturo ${ }^{\circledR}$ (bedaquiline) and Deltyba ${ }^{\circledR}$ (delamanid), novel drugs for multidrug-resistant tuberculosis (MDR-TB), are recommended for diabetes patients when another effective treatment regimen cannot be provided. Currently, there are no clinical data related to the use of these agents in diabetes patients. Possible alterations in the pharmacokinetics of these novel drugs induced by changes in subcutaneous adipose blood flow, gastric emptying, or nephropathy in diabetes patients, and possible drug-drug interactions with hypoglycemic agents, are of special interest, since the efficacy of bedaquiline and delamanid is concentration dependent. Moreover, it is of fundamental importance to avoid possible additive or synergistic effects of adverse drug reactions in this already vulnerable patient group. We reviewed clinical particularities related to the use of bedaquiline and delamanid in patients with type 1 and 2 diabetes mellitus (DM), as well as pharmacological aspects of the concurrent use of these agents with oral and injectable hypoglycemic agents. Bedaquiline shares liver metabolic pathways with several oral hypoglycemic agents, whereas delamanid may compete with several oral hypoglycemic agents and insulin analogs at protein-binding sites. Special concern exists regarding the use of bedaquiline and delamanid in diabetes patients aged $>65$ years and patients with severe renal or hepatic impairment or electrolyte disturbances. Concurrent use of bedaquiline and delamanid with insulin analogs, and other hypoglycemic agents that prolong the heart rate-corrected QT interval, such as sulfonylureas and glinides, may enhance this adverse reaction. Hepatic-related adverse reactions may develop more frequently when these drugs are combined with thiazolidinediones and acarbose. Data from Phase III and postmarketing studies are needed to elucidate the effect of DM and hypoglycemic agents on bedaquiline and delamanid effects in MDR-TB patients.
\end{abstract}

Keywords: bedaquiline, delamanid, oral hypoglycemic agents, insulin analogs, pharmacokinetics

\section{Introduction}

Diabetes mellitus (DM) is an established risk factor for active tuberculosis (TB). An estimated $15 \%-25 \%$ of active TB cases globally are attributable to diabetes. ${ }^{1} \mathrm{DM}$ triples the risk of developing active TB, since it impairs host defenses. Moreover, evidence exists that insulin dependence is an independent predictor of an increased TB risk, and that compared to type 2 diabetes, type 1 diabetes confers a greater risk of contracting TB. ${ }^{2,3}$ Patients with concurrent DM have worse TB treatment outcomes, are 4 times more likely to relapse following TB treatment, and have a higher risk of death from TB than do patients with TB alone. ${ }^{4}$

Multidrug-resistant tuberculosis (MDR-TB) is caused by Mycobacterium tuberculosis resistant to rifampicin and isoniazid. Salindri et al found that type 2 DM was associated with an increased risk of primary MDR-TB, and among 52 patients 
with MDR-TB, the rate of sputum culture conversion was lower in those with diabetes. ${ }^{5}$ Although the biological basis for this observation remains unclear, there is growing evidence of increased MDR-TB prevalence among patients with DM than among those without DM. ${ }^{6,7}$ Kang et al found that in patients undergoing MDR-TB treatment for 8-11 years, DM was independently associated with an increased risk of both treatment failure and death. ${ }^{8}$

Sirturo $^{\circledR}$ (bedaquiline) and Deltyba ${ }^{\circledR}$ (delamanid), new agents for MDR-TB approved by the US Food and Drug Administration in 2012 and 2014, respectively, represent a potentially exciting advancement in MDR-TB therapy. Bedaquiline is a diarylquinoline antimycobacterial drug that inhibits mycobacterial adenosine triphosphate (ATP) synthase, whereas delamanid inhibits the synthesis of mycobacterial cell wall components, methoxymycolic acid, and ketomycolic acid. Both are indicated as part of combination therapy in adults with pulmonary MDR-TB. ${ }^{9,10}$ There are no current trial data related to the provision of bedaquiline and delamanid to diabetes patients with pulmonary manifestation of MDR-TB. However, provisional guidelines from the Centers for Disease Control and Prevention include their use on a case-by-case basis for this and other vulnerable patient groups when an effective treatment regimen cannot otherwise be provided. ${ }^{11}$ An interim policy guidance of the World Health Organization (WHO) states that

as currently there are no data on the efficacy and safety of bedaquiline and delamanid in patients with co-morbid conditions such as DM, liver and/or renal dysfunction, malignancies, alcohol and substance use, careful screening for these conditions prior to treatment initiation is required. ${ }^{12,13}$

Antituberculosis (anti-TB) treatment is affected by DM and hypoglycemic agents; however, glucose control in DM is affected by both TB and some anti-TB drugs (such as rifampicin, which increases the metabolism of most antidiabetic drugs). ${ }^{14}$ Therefore, the treatment of patients affected by both diseases, especially MDR-TB, can be pharmacologically challenging. The aim of this paper was to review all clinical and pharmacological data relevant for the concurrent use of bedaquiline/delamanid and oral hypoglycemic agents/insulin and potentially identify optimal combinations of these agents in terms of patient safety.

\section{Pharmacokinetics: use of bedaquiline and delamanid in diabetes patients} Pharmacokinetic alterations in DM

Clinical experience with bedaquiline and delamanid is too limited to provide data related to the effect of DM on their pharmacology. However, DM may affect the pharmacokinetics of different drugs, including those used to treat TB. It has been suggested that DM may affect absorption (owing to changes in subcutaneous adipose blood flow, muscle blood flow, and gastric emptying), distribution (owing to nonenzymatic albumin glycation), biotransformation (owing to enzyme/transporter regulation involved in this process), and drug excretion (owing to nephropathy). ${ }^{15}$ Nijland et al showed that exposure to rifampicin was strongly reduced in patients with TB and DM, ${ }^{16}$ whereas Kumar et al found that the peak serum concentration $\left(C_{\max }\right)$ and area under the curve $(0-8 \mathrm{~h})$ of pyrazinamide were lower in TB patients with DM than in nondiabetics. ${ }^{17}$ In a preclinical study by Karbownik et al, plasma concentrations of the anticancer drug erlotinib and its metabolites markedly increased in diabetic than in nondiabetic rabbits. ${ }^{18}$ Moreover, data also suggest that DM-related pharmacokinetic alterations of a particular drug cannot be translated to other drugs, which is why it is important to study the effect of each drug and not to draw generalized conclusions. ${ }^{15}$

\section{Bedaquiline and delamanid with oral hypoglycemic agents}

Bedaquiline is administered orally. In vitro and in vivo studies have shown that the metabolism of bedaquiline relies on N-demethylation catalyzed by the CYP3A4 isoenzyme forming the N-monodesmethyl metabolite (M2). ${ }^{9} \mathrm{M} 2$ is the major circulating metabolite, but it seems that in TB-infected patients, the majority of the bactericidal efficacy is achieved by an unchanged drug, as the exposure to M2 in humans is about 4- to 5-fold lower than that of bedaquiline. ${ }^{19}$ Both bedaquiline and M2 show concentration-dependent bactericidal effects. ${ }^{19}$ In addition to CYP3A4, the CYP isoforms $1 \mathrm{~A} 1,2 \mathrm{C} 8$, and $2 \mathrm{C} 18$ also play a role in the metabolism of bedaquiline in vitro (defined as a metabolic rate of $>10 \%$ of that observed with CYP3A4). ${ }^{20}$

CYP450-based drug-drug interaction studies carried out with bedaquiline included known CYP3A4 inducers/ inhibitors and anti-TB drugs: rifampin, ketoconazole, isoniazid/pyrazinamide, nevirapine, lopinavir/ritonavir, ethambutol, kanamycin, pyrazinamide, ofloxacin, and cycloserine. ${ }^{9}$ Significant alterations in bedaquiline plasma concentrations were observed with rifampin and ketoconazole, which is why the concomitant use of these drugs should be avoided for periods of $>14$ days. ${ }^{9}$

Delamanid is metabolized by albumin and, to a lesser extent, CYP3A4. ${ }^{21}$ Therefore, CYP450-based interactions with other drugs are not anticipated. However, it was shown that the interaction of delamanid with the strong CYP3A4 
inducer, rifampicin, reduced exposure to delamanid by $47 \%$ in healthy volunteers, ${ }^{22}$ which is why the European Medicines Agency declared the coadministration of delamanid with strong CYP3A4 enzyme inducers to be contraindicated. According to in vitro data, clinically relevant interactions between delamanid and drugs whose disposition relies on ATP-binding cassette (ABC) transporters, breast cancer resistant proteins (BCRP/ABCG2), solute carrier (SLC) transporters, organic anion transporting polypeptides, or organic cation transporter 1 are improbable. ${ }^{23,24}$ However, the entire metabolic profile of delamanid has not yet been clarified, and the potential for drug interactions with other coadministered medications is a logical concern. ${ }^{10}$

Pharmacokinetic studies of the concurrent use of hypoglycemic agents and bedaquiline/delamanid have not been performed, since other drugs that belong to this group, except bromocriptine, do not show significant potential to inhibit or induce the CYP3A4 enzyme. However, bedaquiline and to a lesser extent delamanid share the same metabolic pathway with certain antidiabetic drugs, and in conditions of infection and inflammation, changes in CYP450 expression and/or activity in the liver and epithelial cells of the small intestine may modulate the pharmacokinetics of both drug groups. ${ }^{25}$ Various studies show that interferons and pro-inflammatory cytokines can downregulate P450 expression in vivo and in hepatocyte cultures, and these mediators are recognized as the main cause of P450 downregulation in infectious/ inflammatory settings. ${ }^{26-28}$ In this context, the logical clinical concern is that patients on a stable drug regimen with oral hypoglycemic agents, who experience a severe infection, would have a decreased exposure to active metabolites of bedaquiline and, to a lesser extent, delamanid, and/or experience hypoglycemic episodes owing to increased blood concentrations of oral antidiabetic drugs. Oral hypoglycemic agents that share the Phase I metabolic pathway with bedaquiline/delamanid are shown in Table 1.

Although during in vitro studies with human liver microsomes and hepatocytes bedaquiline showed little or no potential for inducing/inhibiting major CYP450 isoenzymes, its metabolite, M2, showed marked inhibitory effects on CYP3A4, 2B6, 2D6, and 2C19. ${ }^{29}$ The clinical implications of this finding are unknown. There are no peer-reviewed published data on the potential for pharmacokinetic drug interactions of bedaquiline/delamanid at the level of P-glycoprotein (ABCB1). ${ }^{22}$ At concentrations approximately 10 times higher than those marked as clinically relevant $(\sim 55 \mu \mathrm{g} / \mathrm{mL})$, both bedaquiline and M2 substantially inhibited this transporter essential for the transport of sulfonylureas (SU) and sodium-glucose co-transporter 2 (SGLT-2) inhibitors. ${ }^{29}$
Table I CYP3A4 and/or CYP2C8 crossed metabolic pathways

\begin{tabular}{|c|c|c|}
\hline \multirow[t]{2}{*}{ Oral hypoglycemic agent } & \multicolumn{2}{|c|}{ Novel antituberculosis drug } \\
\hline & $\begin{array}{l}\text { Sirturo }^{\circledR} \\
\text { (bedaquiline) }\end{array}$ & $\begin{array}{l}\text { Deltyba }^{\circledR} \\
\text { (delamanid) }\end{array}$ \\
\hline \multicolumn{3}{|l|}{ Meglitinides } \\
\hline Starlix ${ }^{\circledR}$ (nateglinide) & $3 \mathrm{~A} 4$ & $3 \mathrm{~A} 4$ \\
\hline Prandin $^{\circledR}$ (repaglinide) & $3 \mathrm{~A} 4 / 2 \mathrm{C} 8$ & $3 \mathrm{~A} 4$ \\
\hline \multicolumn{3}{|l|}{ Thiazolidinediones } \\
\hline Actos $^{\circledR}$ (pioglitazone) & $3 \mathrm{~A} 4 / 2 \mathrm{C} 8$ & $3 \mathrm{~A} 4$ \\
\hline Avandia $^{\circledR}$ (rosiglitazone) & $2 \mathrm{C} 8$ & NC \\
\hline \multicolumn{3}{|l|}{ Sulfonylureas } \\
\hline Glyburide $^{\circledR}$ (glibenclamide) & $3 \mathrm{~A} 4$ & $3 \mathrm{~A} 4$ \\
\hline Diamicron $^{\circledR}$ (gliclazide) & $2 \mathrm{C} 8$ & NC \\
\hline Glurenorm $^{\circledR}$ (gliquidone) & $3 \mathrm{~A} 4$ & $3 \mathrm{~A} 4$ \\
\hline \multicolumn{3}{|l|}{ DPP-IV inhibitors } \\
\hline Januvia ${ }^{\circledR}$ (sitagliptin) & $3 \mathrm{~A} 4 / 2 \mathrm{C} 8$ & $3 \mathrm{~A} 4$ \\
\hline Onglyza ${ }^{\circledR}$ (saxagliptin) & $3 \mathrm{~A} 4$ & $3 \mathrm{~A} 4$ \\
\hline \multicolumn{3}{|l|}{ Other } \\
\hline Cycloset $^{\circledR}$ (bromocriptine mesylate) & $3 \mathrm{~A} 4$ & $3 \mathrm{~A} 4$ \\
\hline
\end{tabular}

Abbreviations: NC, no crossing; DPP-IV, dipeptidyl peptidase IV.

The interaction of principal transporters, OATP1B1, OCT1, and OCT2, studied in relation to oral antidiabetics with bedaquiline remains unknown. ${ }^{30}$

Bedaquiline and delamanid are both highly bound $(>99 \%)$ to plasma proteins; $;, 10$ therefore, coadministered drugs may compete for the same plasma protein-binding sites and affect the free drug concentration. This may be the case with their concurrent use with SU (protein binding $>99 \%$ ), glinides (>98\%), or SGLT-2 inhibitors $(>98 \%)$. The pharmacokinetic and clinical consequences of potential proteinbinding displacement remain to be elucidated.

Bromocriptine mesylate, approved as an adjunct therapy to diet and exercise to improve glycemic control in adults with type $2 \mathrm{DM}$, is both a substrate and a very potent inhibitor of CYP3A4 $(\mathrm{Ki}=1.6 \mu \mathrm{M}) .{ }^{31}$ Although there are no studies to confirm this hypothesis, it is logical to conclude that drugs whose clearance is mediated by CYP3A4, such as bedaquiline, should be combined with bromocriptine with special caution. Kim et al showed that glibenclamide also inhibits human CYP3A4 in human liver microsomes, but to a lesser extent than other CYP3A4 inhibitors. ${ }^{32}$ The inhibitory effect of gliquidone on CYP3A4 has also been shown, but the extent of inhibition is unknown. ${ }^{33}$ Colesevelam, a non-absorbed, lipid-lowering agent approved for use as adjunctive therapy in patients with type 2 DM, affects the bioavailability of $>100$ medicinal products, significantly reducing their absorption. ${ }^{34}$ Therefore, the efficacy of both bedaquiline and delamanid may be severely affected by this, since it is concentration dependent.

The plasma concentrations and efficacy of oral hypoglycemic agents and their safety profiles may be significantly affected by the genetic polymorphisms of CYP450 drug-metabolizing 
enzymes, organic cation transporters, or organic aniontransporting polypeptides..$^{35,36}$ However, as a large portion of the pharmacokinetic information of bedaquiline and delamanid remains unclear, it is unknown whether any of these variants influence their metabolism. The only genetic polymorphism that may possibly be relevant for the concurrent use of bedaquiline and delamanid and oral hypoglycemic agents is CYP3A4*18, which plays a major role in the interindividual variability in the pharmacokinetics of repaglinide. ${ }^{37}$

Metformin is an oral hypoglycemic agent that is not metabolized via CYP450 and apparently does not use P-glycoprotein for its transport. There are various preclinical studies showing that metformin may interact pharmacokinetically with many drugs. ${ }^{38,39}$ However, in humans, metformin is cleared from the body by tubular secretion and excreted unchanged in the urine. As metformin is not metabolized, the possibilities of pharmacokinetic drug-drug interactions are minimal; therefore, this hypoglycemic agent could be a key compound for combination with bedaquiline and delamanid.

Studies have suggested that metformin suppresses the inflammatory response by nuclear factor $\kappa \mathrm{B}$ inhibition via adenosine monophosphate (AMP)-activated protein kinase-dependent and -independent pathways, ${ }^{40,41}$ and that metformin anti-inflammatory properties are exerted irrespective of DM status. ${ }^{42}$

Besides its favorable pharmacokinetic profiles, these metformin characteristics make it very suitable for use in severe infections, such as MDR-TB.

\section{Bedaquiline and delamanid with insulin analogs}

For MDR-TB cases with diabetes type 1 and 2, the American Association of Clinical Endocrinologists recommends the use of insulin analogs, as their action is more predictable, resulting in reduced hypoglycemia. Once the disease starts settling, oral hypoglycemic agents may be used. ${ }^{43}$ In severe infections, the positive effects of insulin administration are possibly a result of its anti-inflammatory, vasodilatory, and antioxidant effects, as well as its ability to inhibit platelet aggregation and lipolysis. ${ }^{44}$ In MDR-TB, insulin requirements are initially high, but decrease several weeks after glucotoxicity and the infection have settled. ${ }^{45}$ The pharmacology of exogenous insulin may be altered by the response to infection and concurrent administration of different drugs.

A limited amount of exogenous insulin is metabolized by the liver in its first pass; however, the main organ responsible for metabolizing exogenous insulin administered to diabetes patients $(\sim 80 \%)$ is the kidney. ${ }^{46-48}$ Approximately $65 \%$ of insulin is filtered in the glomerulus and metabolized in the cells of the proximal tubule. The remaining insulin $(\sim 35 \%)$ diffuses from post-glomerular peritubular vessels to the contraluminal cell membrane of the proximal tubular cell, where it is also degraded. ${ }^{49}$ Although insulin analogs are also metabolized in the liver, they do not share the same metabolic pathways as bedaquiline and delamanid, so the hepatic alteration of the pharmacokinetic parameters of these drugs is not expected.

In general, information about interactions between insulin and other medications is relatively limited; the summaries of product characteristics of the insulin analogs do not include specific drug interactions. It has been shown that many drugs potentially increase the hypoglycemic effect of insulin (oral antidiabetic products, pramlintide, angiotensin-converting enzyme inhibitors, disopyramide, fibrates, fluoxetine, monoamine oxidase inhibitors, propoxyphene, pentoxifylline, salicylates, somatostatin analogs, sulfonamide antibiotics) and reduce it (corticosteroids, niacin, danazol, diuretics, sympathomimetic agents, glucagon, isoniazid, phenothiazine derivatives, somatropin, thyroid hormones, estrogens, progestogens, protease inhibitors, atypical antipsychotic medications), ${ }^{50-54}$ but the mechanisms responsible for these effects are mostly unknown. Currently, there are no data related to potential hypoglycemic or hyperglycemic properties of bedaquiline or delamanid which may affect the hypoglycemic potential of insulin analogs.

Following subcutaneous injection, insulin detemir and degludec extensively bind to albumin. At a steady state, the concentration of unbound insulin is considerably reduced, leading to more stable plasma insulin levels. ${ }^{55,56}$ The prolonged action of detemir and degludec, achieved by slow absorption from the subcutaneous depot, appears to be partially mediated by binding to albumin via a fatty acid chain. ${ }^{57}$ Limited data are available in humans related to the relationship between the pharmacodynamic and pharmacokinetic properties of detemir and degludec and the plasma level of albumin. However, potential interactions of detemir and degludec with bedaquiline and delamanid should be considered.

Positive and negative aspects of the use of insulin analogs and metformin in diabetes patients with MDR-TB are summarized in Table 2 .

\section{Bedaquiline and delamanid with GLP-I receptor agonists and pramlintide}

The potential of glucagon-like peptide-1 (GLP-1) receptor agonists and pramlintide to delay gastric emptying may 
Table 2 Advantages and disadvantages of the use of insulin analogs and metformin in multidrug-resistant tuberculosis patients treated with bedaquiline/delamanid

\begin{tabular}{|c|c|c|}
\hline Clinical impact & Metformin & Insulin analogs \\
\hline \multirow[t]{6}{*}{ Advantages } & $\begin{array}{l}\text { No interaction at the } \mathrm{ABCBI}, \mathrm{CYP} 3 \mathrm{~A} 4 \text {, or protein- } \\
\text { binding site levels }\end{array}$ & No interaction at the transporter or CYP3A4 levels \\
\hline & & Possible interaction at the protein-binding site (detemir, degludec) \\
\hline & Anti-inflammatory properties & Anti-inflammatory, vasodilatory, and antioxidant properties \\
\hline & No risk of QT prolongation & No risk of $\mathrm{QT}$ prolongation \\
\hline & & $\begin{array}{l}\text { More predictable hypoglycemic effect in severe infection } \\
\text { compared to metformin }\end{array}$ \\
\hline & & Allowed in renal and hepatic impairment \\
\hline \multirow[t]{3}{*}{ Disadvantages } & Enhanced gastrointestinal toxicity & \\
\hline & Unpredictable hypoglycemic effect in severe infections & $\begin{array}{l}\text { Possibly increased risk of hypertensive episodes and muscle } \\
\text { damage (glargine) }\end{array}$ \\
\hline & Not allowed with renal or hepatic impairment & \\
\hline
\end{tabular}

reduce the extent and rate of absorption of many drugs administered per os. ${ }^{58}$ Patients should be advised to take those medicinal products whose efficacy particularly depends on threshold plasma concentrations, such as bedaquiline and delamanid, at least $1 \mathrm{~h}$ before the injection of GLP-1 receptor agonists or pramlintide..$^{58}$

Liraglutide is the only drug from this group which may possibly interact with bedaquiline/delamanid at the proteinbinding level, as it is highly protein-bound $(>98 \%) .{ }^{59}$ Either the GLP-1 receptor agonists undergoes minimal systemic metabolism or they are endogenously metabolized by dipeptidyl peptidase IV (DPP-IV) and endogenous endopeptidases ${ }^{58}$ whereas pramlintide is metabolized primarily by the kidneys, ${ }^{60}$ so other pharmacokinetic interactions are not expected with bedaquiline and delamanid. Potential pharmacokinetic interactions of bedaquiline/delamanid and hypoglycemic agents with the resulting clinical effects are summarized in Table 3.

\section{Pharmacodynamics: use of bedaquiline and delamanid in diabetes patients Mechanism of action and pharmacodynamic interactions}

Bedaquiline inhibits mycobacterial ATP synthase, an essential enzyme for energy generation in M. tuberculosis. ${ }^{9,61}$ Since its mechanism of action differs from those of other antimycobacterial agents, bedaquiline has the capacity to retain efficacy against certain $M$. tuberculosis isolates that are resistant to other drugs. Its minimal inhibitory concentration (MIC) for drug-sensitive and drug-resistant strains ranges from $\leq 0.008$ to $0.12 \mathrm{mg} / \mathrm{L},{ }^{9}$ but these data have not yet been validated in human studies. The intracellular bactericidal effect of bedaquiline in macrophages and a macrophagelike cell line was greater than its extracellular activity., ${ }^{9,61}$ Delamanid inhibits the synthesis of the mycobacterial cell

Table 3 Possible pharmacokinetic interactions between bedaquiline/delamanid and hypoglycemic agents and their expected clinical effects

\begin{tabular}{|c|c|c|}
\hline Interaction level & Possible pharmacokinetics & Expected clinical effects \\
\hline Transporter level (ABCBI) & Inhibition of SU and SGLT-2 inhibitors & Lack of hypoglycemic efficacy \\
\hline Protein-binding level & $\begin{array}{l}\text { Competition for protein-binding sites (bedaquiline/delamanid vs SU, } \\
\text { glinides, SGLT-2 inhibitors) }\end{array}$ & Unclear \\
\hline \multirow[t]{4}{*}{ Hepatic metabolism level } & $\begin{array}{l}\text { Decreased exposure to bedaquiline/delamanid with strong CYP3A4 } \\
\text { inducers (bromocriptine) }\end{array}$ & Lack of antituberculosis efficacy \\
\hline & $\begin{array}{l}\text { Increased exposure to oral hypoglycemic agents mainly metabolized by } \\
\text { CYP3A4 (nateglinide, sitagliptin, saxagliptin) due to inhibitory effect of M2 }\end{array}$ & Hypoglycemic episodes \\
\hline & $\begin{array}{l}\text { Increased exposure to bedaquiline in severe infection (downregulation } \\
\text { of P450 expression) }\end{array}$ & Toxicity of bedaquiline \\
\hline & $\begin{array}{l}\text { Increased exposure to oral hypoglycemic agents in severe infections } \\
\text { (downregulation of P450 expression) }\end{array}$ & Hypoglycemic episodes \\
\hline
\end{tabular}

Note: $A B C B I$ is a $P$-glycoprotein, and $M 2$ is the bedaquiline metabolite.

Abbreviation: $\mathrm{SU}$, sulfonylureas. 
wall components methoxymycolic and ketomycolic acid. ${ }^{10,24}$ Susceptibility testing breakpoints of delamanid have not yet been determined.

Clinical studies exploring the effect of diabetes on the pharmacodynamics of drugs are still very limited. Previously published studies have reported the effects of diabetes on the drug dose-response of cardiovascular drugs, such as lipidlowering agents ${ }^{62-64}$ and antihypertension drugs, ${ }^{65}$ but it is unclear whether these studies show true pharmacodynamic changes or merely alterations in pharmacokinetics.

Clinical studies performed with bedaquiline and delamanid did not specifically address the dose/concentrationeffect relationship between bedaquiline/delamanid and other drugs at the receptor, signaling, or effector level. ${ }^{9,10,21,66,67}$ However, it has been observed that the use of bedaquiline and delamanid with QT-prolonging medications increases the risk of prolonged QT by additive or synergistic mechanisms, probably due to co-inhibition of the ATP-dependent $\mathrm{K}^{+}$channels. ${ }^{9,10}$ Currently, there is no clinical evidence that the hypoglycemic agents could provoke similar effects. Nonetheless, some antidiabetics, such as SU and glinide agents, delay repolarization times by the same mechanism. ${ }^{68}$ Therefore, combining these 2 drug groups with bedaquiline/ delamanid requires caution.

\section{Mechanisms of resistance}

Resistance to bedaquiline and delamanid has been documented during treatment. Acquired resistance mechanisms that affect bedaquiline MICs include strain mutations in the $a t p E$ gene, which codes for ATP synthase, ${ }^{69}$ and in the $R v 0678$ gene, which regulates the expression of the MmpS5-MmpL5 efflux pump. ${ }^{70-72}$ The latter also confers cross-resistance to clofazimine. ${ }^{70}$ Target-based mutations generated in preclinical studies led to 8- to 133-fold increases in bedaquiline MICs, whereas efflux-based mutations, seen in preclinical and clinical isolates, led to 2- to 8-fold increases in bedaquiline MICs. ${ }^{9,71,72}$ Delamanid requires activation by mycobacterial F420-dependent deazaflavin-dependent nitroreductase coenzymes. Mutation in 1 of the 5 coenzyme $F 420$ genes ( $f g d, R v 3547, f b i A, f b i B$, and $f b i C$ ) is suggested as the mechanism for resistance against delamanid in mycobacteria. ${ }^{10,22}$ In 2015, Bloemberg et al showed that a mutation in $m m p R$ was associated with bedaquiline resistance, whereas 2 mutations in $f b i A$ and $f g d l$ were associated with resistance to delamanid. ${ }^{73}$

Almeida et al found that the loss of function of the $R v 2535$ gene, which codes for putative Xaa-Pro aminopeptidase, leads to reduced susceptibility both in vitro and in mice. ${ }^{74}$
There is no evidence of host genome mutations related to bedaquiline/delamanid resistance.

\section{Clinical efficacy and safety}

Evidence of bedaquiline's safety and efficacy came from 2-stage Phase II, randomized, placebo-controlled trials. .,66,75 $^{9}$ Investigators included individuals with positive sputum smears and sensitivity to at least 3 of the 5 classes of drugs used in the background antimycobacterial regimen (BR) for pulmonary MDR-TB. The primary outcome parameter was the time to sputum culture conversion during treatment with bedaquiline or placebo. In the first stage, 47 patients were randomly assigned to receive 8 weeks of placebo or bedaquiline ( $400 \mathrm{mg}$ daily for 2 weeks, followed by $200 \mathrm{mg}$ 3 times weekly for 6 weeks) in addition to the BR. Adding bedaquiline to the $\mathrm{BR}$ reduced the time to sputum culture conversion (hazard ratio [HR] 11.8, confidence interval [CI] 2.3-61.3, $P=0.003$ ) and increased the proportion of patients whose sputum cultures converted to negative $(48 \%$ [10 of 21$]$ vs $9 \%$ [2/23] at 8 weeks, $P=0.003)$. In the second stage, 160 patients were randomized to receive bedaquiline or a placebo $400 \mathrm{mg}$ daily for 2 weeks followed by $200 \mathrm{mg}$ thrice weekly for 22 weeks. The time to sputum culture conversion was again significantly reduced in the bedaquiline group (HR 2.44, CI 1.57-3.80, $P<0.0001$ ). ${ }^{9,66,75}$ Headaches ( $24 \%$ vs $11 \%$ ), nausea (35\% vs $26 \%$ ), and arthralgias ( $29 \%$ vs $20 \%$ ) were more common in the bedaquiline group. More patients in the bedaquiline group than in the placebo group died (2/81 patients in the placebo group, in contrast with 10 of 79 bedaquiline-treated patients). The unexpected imbalance in deaths between the 2 treatment groups remained unexplained. ${ }^{9,66,75}$

A single-arm, Phase II trial involving 233 patients, who were sputum smear positive within 6 months prior to screening, evaluated the safety, tolerability, and efficacy of 24-week treatment with open-label bedaquiline as part of an individualized treatment regimen. ${ }^{71}$ The median time to sputum culture conversion was consistent with that found in the second stage of the controlled study. Mean increases from baseline values in the heart rate-corrected QT (QTc) were observed both in controlled and uncontrolled trials. , $^{9,66,71,75}$ The largest mean increase from baseline values in QTc in the second phase of the controlled trial was $15.7 \mathrm{~ms}$ (at week 18), whereas the maximal mean QTc increase in the uncontrolled trial was $23.7 \mathrm{~ms}$. The QTc returned to normal after discontinuation of bedaquiline.

The safety, tolerability, and efficacy of an 8-week treatment with delamanid in combination with the 
WHO-recommended BR were evaluated in a multicenter, double-blind, placebo-controlled study. ${ }^{10,21}$ The authors assigned 481 patients with pulmonary MDR-TB to receive delamanid at a dose of $100 \mathrm{mg}$ twice daily (161 patients) or $200 \mathrm{mg}$ twice daily (160 patients), or placebo (160 patients). Higher sputum culture conversion rates were observed in the groups that received $100 \mathrm{mg}$ twice-daily and $200 \mathrm{mg}$ twice-daily treatment compared to patients on placebo (45.4\% vs $29.6 \%, P=0.008$ and $41.9 \%$ vs $29.6 \%, P=0.04$, respectively). ${ }^{10,21}$

The most frequently observed adverse drug reactions in patients treated with delamanid (incidence $>10 \%$ ) were nausea (38.3\%), vomiting (33\%), and dizziness (30.2\%). QT prolongation was reported significantly more frequently in the groups that received delamanid. ${ }^{10,21}$

\section{Clinical considerations related to the use of bedaquiline and delamanid in diabetes patients Aging}

Aging is associated with structural and functional changes affecting drug pharmacokinetics and pharmacodynamics. Besides impaired renal and hepatic functions, other factors, such as reduced gastric acid secretion, ${ }^{76,77}$ gastric emptying, ${ }^{78}$ reduced splanchnic blood flow, reduced absorptive capacity of the small intestine, reduction in liver mass and blood flow, changes in body composition, ${ }^{79}$ and increased sensitivity to drugs, may lead to important changes in plasma concentrations. Eleven Phase I trials with bedaquiline $(\mathrm{N}=265)$, and 1 Phase IIa $(\mathrm{N}=47)$ and 2 Phase IIb trials $(\mathrm{N}=393)$ that included patients with drug-susceptible TB did not include sufficient numbers of patients aged $\geq 65$ years to determine whether their response to these drugs is different from that of younger patients. ${ }^{80}$ Delamanid was investigated through 12 Phase I trials $(\mathrm{N}=887)$ and 1 Phase II trial $(\mathrm{N}=481)$, but no data are available for elderly patients. ${ }^{10}$ As the population of patients affected by type $2 \mathrm{DM}$ predominantly belongs to this age group, the therapeutic effects and safety profiles of these drugs in this population remain unclear.

\section{Renal impairment}

About one-third of patients with type $1 \mathrm{DM}$ and 10\%-40\% of those with type $2 \mathrm{DM}$ will eventually suffer from renal failure. Mild renal impairment $(50-80 \mathrm{~mL} / \mathrm{min}$ creatinine clearance [CrCLN]) does not appear to affect bedaquiline/ delamanid exposure, as their renal excretion is not substantial $\left(<0.001 \%\right.$ for bedaquiline and $<5 \%$ for delamanid). ${ }^{9,10}$
In a population pharmacokinetic analysis of TB patients treated with bedaquiline ( $200 \mathrm{mg} 3$ times a week), CrCLN did not affect the pharmacokinetic parameters of bedaquiline. ${ }^{9}$ However, in diabetes patients with severe renal impairment or in patients who required hemodialysis or peritoneal dialysis, drug concentrations could be elevated owing to renal dysfunction-mediated alteration of drug pharmacokinetics. Although the Summary of Product Characteristics (SmPC) for bedaquiline advises that this drug should be used with caution in patients with severe renal impairment $(<30 \mathrm{~mL} / \mathrm{min})$, the SmPC for delamanid discourages its use in this patient group. ${ }^{9,10}$

Diabetic kidney disease associated with micro- or macroalbuminuria represents another significant challenge, as both drugs extensively bind to plasma proteins ( $>99.9 \%$ for bedaquiline and $>99.5 \%$ for delamanid). ${ }^{9,10}$ Additionally, as delamanid is primarily metabolized in plasma by albumin, hypoalbuminemia may lead to increased blood concentrations of this drug. In a clinical study with delamanid, hypoalbuminemia was associated with an elevated risk of the prolongation of the QTc interval in the delamanid treatment group owing to increased plasma levels of this drug. ${ }^{10,21}$ Therefore, delamanid is contraindicated in patients with albumin $<2.8 \mathrm{~g} / \mathrm{dL}$. ${ }^{10,21}$

\section{Hepatic impairment}

The entire spectrum of liver disease (abnormal liver enzymes, nonalcoholic fatty liver disease, cirrhosis, hepatocellular carcinoma, acute liver failure) is seen in patients with type 2 DM. ${ }^{21}$ It seems that type $1 \mathrm{DM}$ is not associated with fat accumulation in the liver if glucose levels are well controlled, but type 2 DM may have a $70 \%$ correlation, regardless of glycemic control. ${ }^{81}$ Nonalcoholic fatty liver disease-associated alterations of liver function and transporter expression can provoke dramatic changes in drug disposition. ${ }^{82}$ Acute hepatic failure affects drug metabolism through changes in hepatic blood flow, liver enzyme activity, and the binding of drugs to plasma proteins. ${ }^{83}$ It appears that the pharmacokinetics of bedaquiline and delamanid does not change after single-dose administration to subjects with moderate hepatic impairment (Child-Pugh B score). ${ }^{9,10}$ However, neither of these agents has been studied in patients with severe hepatic impairment, and the SmPCs of both bedaquiline and delamanid discourage their use in this patient group. ${ }^{9,10}$

\section{Hypokalemia}

Diabetes patients often present electrolyte disturbances, including hypokalemia resulting from insulin administration, 
and gastrointestinal or renal loss of $\mathrm{K}^{+}$. Insulin can induce hypokalemia because it promotes the entry of $\mathrm{K}^{+}$into skeletal muscles and hepatic cells by increasing the activity of the $\mathrm{Na}^{+} / \mathrm{K}^{+}$-ATPase pump. ${ }^{84}$ The increased epinephrine secretion caused by insulin-induced hypoglycemia could play a contributory role. ${ }^{85}$ Special precautions should be taken when delamanid is administered to patients with hypokalemia, as it frequently induces this electrolyte disturbance itself $(\geq 1 / 10){ }^{10}$

Low potassium levels may potentiate the development of QTc prolongation that commonly appears with bedaquiline and delamanid. The WHO expert group calls for strict monitoring of potassium imbalances in patients receiving these drugs. ${ }^{12,13}$

\section{Phospholipidosis}

Bedaquiline and delamanid have been shown to induce phospholipidosis at most doses and exposures in drug-treated animals. ${ }^{9,10}$ The possible functional implications of this condition upon cellular or tissue function are not explained. However, this finding may be of special interest for diabetes patients, as there are reports suggesting the role of druginduced phospholipidosis in progressive renal insufficiency in humans. ${ }^{86}$ Moreover, several animal models and human studies indicate that the formation of oxidative phospholipids represents a general mechanism that may play a major role in chronic inflammatory diseases such as DM. ${ }^{87}$

\section{Adverse drug reactions QT interval prolongation}

Considering the long duration of MDR-TB treatment and potential appearance of adverse effects long after the treatment has been completed, particularly given that the terminal elimination of bedaquiline takes 5.5 months (due to its long plasma half-life, high tissue penetration, and long half-life in tissues), ${ }^{9}$ evaluating cardiovascular side effects is particularly important. Both bedaquiline and delamanid, in multiple-dosing regimens, can prolong the QT interval. ${ }^{9,10}$ A prolonged heart rate-corrected QTc interval is defined as above $450 \mathrm{~ms}$ in men and $470 \mathrm{~ms}$ in women, and it is recognized as a well-known risk factor for ventricular arrhythmias and sudden cardiac death. ${ }^{88}$

The risk of QTc prolongation with bedaquiline/delamanid appears to be the highest during the treatment phase, but could extend beyond this phase. It seems that plasma concentrations of the M2 metabolite of bedaquiline correlate with QT interval prolongation. ${ }^{9}$ In addition, the use of bedaquiline and delamanid with QT-prolonging medications increases the risk of prolonged QT by additive or synergistic mechanisms. ${ }^{9}$
For bedaquiline, this effect was observed in studies of its concurrent use with ketoconazole and clofazimine, whereas for delamanid, additive/synergistic QT prolongation was observed with levofloxacin. ${ }^{9,10}$ Therefore, baseline testing and monitoring for QT prolongation and arrhythmia development, especially in combination with other QT-prolonging drugs, are imperative according to the WHO expert group. ${ }^{12,13}$

SU and glinide agents inhibit ATP-dependent $\mathrm{K}^{+}$channels, delaying repolarization times and prolonging the QTc. ${ }^{68}$ As stated earlier, there are no registered episodes of QTc prolongation as a consequence of the concurrent use of bedaquiline/ delamanid and hypoglycemic drugs. However, it appears that fluoroquinolones increase the QTc prolongation risk when used with bedaquiline/delamanid, and have the same potential to block the cardiac voltage-gated potassium channels, particularly the rapid component (IKr) of the delayed rectifier potassium current (IK), prolonging the QTc. ${ }^{89}$ The same mechanism has been proposed for ketoconazole. ${ }^{90}$

As delamanid and bedaquiline are metabolized by CYP3A4, CYP3A4 inhibitors may further prolong the QTc interval, especially the potent CYP3A4 inhibitor bromocriptine. $^{22}$

Regarding other cardiovascular effects, hypotension was commonly seen in clinical studies with delamanid. ${ }^{10}$ Bromocriptine is a hypoglycemic agent that also produces hypotension, and symptomatic orthostatic hypotension can occur in patients treated with bromocriptine mesylate for any indication. ${ }^{31}$ In postpartum studies performed with bromocriptine mesylate, decreases in supine systolic and diastolic pressures have been observed in almost one-third of patients who were treated with this drug. ${ }^{91,92}$ The pathophysiology of delamanid-induced hypotension is not clear yet, but its concurrent use with other potentially hypotensive agents should be closely monitored.

Cardiovascular toxicity is not common among insulin analogs. However, there are numerous case reports describing hypertensive episodes with glargine, although its SmPC does not include hypertension as a possible side effect. ${ }^{54}$ Delamanid has also produced hypertension in $\geq 1 / 100$ to $<1 / 10$ treated patients. ${ }^{10,21}$ Although the causal relationship between hypertension and glargine exposure has not been established yet, monitoring this side effect when combining glargine and delamanid is recommended.

\section{Hepatic-related adverse drug reactions and gastrointestinal disorders}

In Phase IIb clinical studies performed with bedaquiline, aminotransferase elevations of at least 3 times the upper limit of the normal were observed more frequently in the 
bedaquiline treatment group $(11 / 102 ; 10.8 \%)$ than in the placebo treatment group $(6 / 105 ; 5.7 \%))^{9,71}$ The elevation in liver enzyme levels increased gradually over the 24-week treatment period. ${ }^{9,71}$ Therefore, monitoring symptoms and laboratory tests (alanine transaminase, aspartate transaminase, alkaline phosphatase, and bilirubin) at baseline levels and monthly when on treatment with bedaquiline are advised. Hepatobiliary disorders were classified as common $(\geq 1 / 100$ to $<1 / 10$ ) for bedaquiline, whereas in studies with delamanid, they were uncommon $(\geq 1 / 1,000$ to $<1 / 100)$. ${ }^{9,10,21}$ The mechanism of bedaquiline-related hepatotoxicity remains unknown. However, it is advised that a combination of bedaquiline and other hepatotoxic drugs should be avoided. Therefore, special attention should be given to the concurrent use of bedaquiline and thiazolidinediones and acarbose, also known for their hepatotoxic potential..$^{93,94}$

Nausea and vomiting were reported as very common in bedaquiline clinical studies ( $\geq 1 / 10$ ), and diarrhea was classified as a common adverse drug reaction..$^{9,66,71,75}$ Similarly, in the double-blind, placebo-controlled clinical trial with delamanid, vomiting, diarrhea, nausea, and upper abdominal pain were commonly observed $(\geq 1 / 10) .{ }^{10,21}$ These reactions may be potentiated when bedaquiline/delamanid are combined with biguanides, alpha-glucosidase inhibitors, bromocriptine, glinides, and SGLT-2 inhibitors, which also produce gastrointestinal disturbances, similarly to insulin analogs, GLP-1 receptor agonists, and pramlintide.

Pancreatitis was not seen more commonly in clinical trials with bedaquiline than with placebos. ${ }^{9,66,71,75}$ Nonetheless, an increase in pancreatic enzymes was observed in patients taking bedaquiline, although it was rare and the causal relationship was not determined. ${ }^{9}$ Until more information from Phase III clinical studies becomes available, precaution should be taken when bedaquiline is combined with DPP-IV inhibitors, exenatide, and GLP-1 receptor agonists that could produce pancreatitis or alter pancreatic enzymes.

\section{Rhabdomyolysis potential}

Degenerative changes in skeletal muscles were seen in mice, rats, and dogs treated with bedaquiline. ${ }^{13}$ Fibrohistiocytic infiltration and degeneration of muscle fibers in the tongue and quadriceps were detected in rats treated for 13 weeks with bedaquiline at high doses $(24 \mathrm{mg} / \mathrm{kg})$. This myopathy was reversible 12 weeks after the treatment period. ${ }^{13}$ In clinical trials with bedaquiline, there were no reported cases of rhabdomyolysis. ${ }^{9}$ However, myalgia was reported as a common side effect for both bedaquiline and delamanid., ${ }^{9,10}$

Since bedaquiline-induced muscle damage is reversible, the risk of possible muscle effects may not outweigh the benefit that this drug has for MDR-TB treatment. However, combining bedaquiline with other drugs that may cause muscle damage, such as pioglitazone or insulin glargine, may be dangerous. Pioglitazone was reported to cause severe acute rhabdomyolysis, with dose-independent myalgia as a common side effect. ${ }^{95,96}$ Further studies are needed to elucidate bedaquiline's potential for inducing rhabdomyolysis in humans and the effects of its concomitant use with other agents that could potentially induce muscle damage.

\section{Peripheral neuropathy}

Transitory, acute painful peripheral neuropathy has been observed in patients with a rapid improvement in glycemic control with detemir. ${ }^{55}$ Peripheral neuropathy has been reported as a common side effect of delamanid, ${ }^{10}$ but it remains unclear whether the concurrent use of these drugs would aggravate this side effect.

\section{Therapeutic drug monitoring}

Since the antimicrobial action of bedaquiline and delamanid is exposure-dependent, ${ }^{20,97}$ it is necessary that target exposures be ensured. Current preclinical data related to optimal plasma concentrations of these drugs have not yet been translated to and validated in human studies. Therefore, the desired $C_{\max }$ relative to the MIC has not been defined.

In TB settings, therapeutic drug monitoring (TDM) permits the clinician to adjust the therapy in a timely manner. ${ }^{98,99}$ For individuals with no other comorbidities who respond to the standard TB therapeutic options, these adjustments may not be necessary. However, in patients with MDR-TB and concurrent diseases such as DM, the pharmacological profile is significantly complicated owing to altered food intake, malabsorption, altered metabolism, drug-drug interactions, or adverse events. ${ }^{98,99}$ Such patients may benefit from TDM, avoiding mycobacterial exposure to subinhibitory drug concentrations. Moreover, because patients with DM frequently have other comorbidities, checking serum concentrations early may avoid complications and ensure adequate therapy. ${ }^{98}$

DM was found to be significantly associated with slow responses $(P<0.001)$ to TB treatment in the study performed by Heysell et al. ${ }^{100}$ Routine early TDM (at 2 weeks of anti-TB therapy) in diabetes patients showed that $76 \%$ of them had subtherapeutic levels of isoniazid or rifampin or both. ${ }^{100}$ The goal of serum drug concentration testing in diabetics is to make early changes in treatment regimens to improve sputum conversion and diminish the slow response rates. In addition, prolonging the already long treatment with bedaquiline/delamanid ( 24 weeks) may be prevented using plasma concentration measurements. ${ }^{101}$ 


\section{Conclusion}

There is a lack of sufficient pharmacokinetic and clinical data necessary to ensure the safe utilization of bedaquiline and delamanid in patients with DM. This particularly includes diabetes patients aged $>65$ years and those with severe renal and hepatic impairment, as well as patients with hypokalemia. Based on the available data, insulin analogs appear to be safe to combine with bedaquiline and delamanid, although potential insulin-induced hypokalemia should be cautiously monitored, as it could potentially prolong the QTc. Moreover, the anti-inflammatory, vasodilatory, and antioxidant effects of insulin analogs support their use in severe infections, such as MDR-TB. The possibility of additive or synergic QTc prolongation also exists when bedaquiline and delamanid are combined with SU and glinides, since they share the same potential to block the cardiac voltage-gated potassium channels. The CYP3A4 inhibitor, bromocriptine, may prolong the QTc interval owing to potentially increased plasma bedaquiline concentrations. Among oral hypoglycemic drugs, metformin possibly has the most favorable pharmacokinetic and safety profile when combined with bedaquiline and delamanid. Its severe gastrointestinal toxicity may be increased with the concurrent use of these drugs, but considering its anti-inflammatory properties, the benefits of its use in MDR-TB outweigh the risk. For altered drug metabolism, seen in DM and severe infections, the TDM of concentrationdependent drugs, such as bedaquiline and delamanid, may be crucial. Further pharmacokinetic, Phase III clinical, and postmarketing studies are essential in ensuring the safe use of these agents in diabetes patients.

\section{Disclosure}

The authors report no conflicts of interest in this work.

\section{References}

1. Lönnroth K, Roglic G, Harries AD. Improving tuberculosis prevention and care through addressing the global diabetes epidemic: from evidence to policy and practice. Lancet Diabetes Endocrinol. 2014;2(9):730-739.

2. Olmos P, Donoso J, Rojas N, et al. [Tuberculosis and diabetes mellitus: a longitudinal-retrospective study in a teaching hospital]. Rev Med Chil. 1989;117(9):979-983. Spanish.

3. Swai AB, McLarty DG, Mugusi F. Tuberculosis in diabetic patients in Tanzania. Trop Doct. 1990;20(4):147-150.

4. Riza AL, Pearson F, Ugarte-Gil C, et al. Clinical management of concurrent diabetes and tuberculosis and the implications for patient services. Lancet Diabetes Endocrinol. 2014;2(9):740-753.

5. Salindri AD, Kipiani M, Kempker RR, et al. Diabetes reduces the rate of sputum culture conversion in patients with newly diagnosed multidrugresistant tuberculosis. Open Forum Infect Dis. 2016;3(3):ofw126.

6. Hsu AH, Lee JJ, Chiang CY, Li YH, Chen LK, Lin CB. Diabetes is associated with drug-resistant tuberculosis in Eastern Taiwan. Int $J$ Tuberc Lung Dis. 2013;17(3):354-356.
7. Duangrithi D, Thanachartwet V, Desakorn V, et al. Impact of diabetes mellitus on clinical parameters and treatment outcomes of newly diagnosed pulmonary tuberculosis patients in Thailand. Int J Clin Pract. 2013; 67(11):1199-1209.

8. Kang YA, Kim SY, Jo KW, et al. Impact of diabetes on treatment outcomes and long-term survival in multidrug-resistant tuberculosis. Respiration. 2013;86(6):472-478.

9. Sirturo ${ }^{\circledR}$ (delamanid) tablets [prescribing information]. Beerse: JanssenCilag Int. N.V.; 2012.

10. Deltyba ${ }^{\circledR}$ (delamanid) film-coated tablets [prescribing information]. Munchen: Otsuka Novel Products GmbH; 2014.

11. Centers for Disease Control and Prevention. Provisional CDC guidelines for the use and safety monitoring of bedaquiline fumarate (Sirturo) for the treatment of multidrug-resistant tuberculosis. MMWR Recomm Rep. 2013;62(RR-09):1-12.

12. WHO. The use of delamanid in the treatment of multidrug-resistant tuberculosis. Interim policy guidance. 2014. Available from: http://apps. who.int/iris/bitstream/10665/137334/1/WHO_HTM_TB_2014.23_eng. pdf. Accessed August 16, 2016.

13. WHO. The use of bedaquiline in the treatment of multidrug-resistant tuberculosis. Interim policy guidance. 2012. Available from: http:// apps.who.int/iris/bitstream/10665/84879/1/9789241505482_eng.pdf. Accessed August 16, 2016.

14. van Crevel R, Dockrell HM; TANDEM Consortium. Understanding diabetes and tuberculosis. Lancet Diabetes Endocrinol. 2014;2(4): 270-272.

15. Dostalek M, Akhlaghi F, Puzanovova M. Effect of diabetes mellitus on pharmacokinetic and pharmacodynamic properties of drugs. Clin Pharmacokinet. 2012;51(8):481-499.

16. Nijland HM, Ruslami R, Stalenhoef JE, et al. Exposure to rifampicin is strongly reduced in patients with tuberculosis and type 2 diabetes. Clin Infect Dis. 2006;43(7):848-854.

17. Kumar H, Kannan T, Chandrasekaran V, et al. Pharmacokinetics of thrice-weekly rifampicin, isoniazid and pyrazinamide in adult tuberculosis patients in India. Int J Tuberc Lung Dis. 2016;20(9):1236-1241.

18. Karbownik A, Szałek E, Sobańska K, Grabowski T, Wolc A, Grześkowiak E. The alteration of pharmacokinetics of erlotinib and OSI420 in type 1 diabetic rabbits. Pharmacol Rep. 2016;68(5): 964-968.

19. McLeay SC, Vis P, van Heeswijk RP, Green B. Population pharmacokinetics of bedaquiline (TMC207), a novel antituberculosis drug. Antimicrob Agents Chemother. 2014;58(9):5315-5324.

20. Rouan MC, Lounis N, Gevers T, et al. Pharmacokinetics and pharmacodynamics of TMC207 and its N-desmethyl metabolite in a murine model of tuberculosis. Antimicrob Agents Chemother. 2012; 56(3):1444-1451.

21. Gler MT, Skripconoka V, Sanchez-Garavito E, et al. Delamanid for multidrug-resistant pulmonary tuberculosis. N Engl J Med. 2012;366(23): 2151-2160.

22. Lewis JM, Sloan DJ. The role of delamanid in the treatment of drugresistant tuberculosis. Ther Clin Risk Manag. 2015;11:779-791.

23. Sasabe H, Shimokawa Y, Shibata M, et al. Antitubercular agent delamanid and metabolites as substrates and inhibitors of $\mathrm{ABC}$ and solute carrier transporters. Antimicrob Agents Chemother. 2016;60(6): 3497-3508.

24. Field SK. Safety and efficacy of delamanid in the treatment of multidrug-resistant tuberculosis (MDR-TB). Clin Med Insights Ther. 2013;5(5):137-149.

25. Shimada T, Yamazaki H, Mimura M, Inui Y, Guengerich FP. Interindividual variations in human liver cytochrome P-450 enzymes involved in the oxidation of drugs, carcinogens and toxic chemicals: studies with liver microsomes of 30 Japanese and 30 Caucasians. J Pharmacol Exp Ther. 1994;270(1):414-423.

26. Renton KW. Cytochrome $\mathrm{P} 450$ regulation and drug biotransformation during inflammation and infection. Curr Drug Metab. 2004;5(3): 235-243. 
27. Aitken AE, Richardson TA, Morgan ET. Regulation of drug-metabolizing enzymes and transporters in inflammation. Annu Rev Pharmacol Toxicol. 2006;46:123-149.

28. Xu DX, Wang JP, Sun MF, Chen YH, Wei W. Lipopolysaccharide downregulates the expressions of intestinal pregnane $\mathrm{X}$ receptor and cytochrome p450 3a11. Eur J Pharmacol. 2006;536(1-2):162-170.

29. Sirturo ${ }^{\circledR}$ (bedaquiline) tablets [briefing package]. Silver Spring, MD: FDA; 2012. Available from: http://www.fda.gov/downloads/ AdvisoryCommittees/CommitteesMeetingMaterials/Drugs/AntiInfectiveDrugsAdvisoryCommittee/UCM329258.pdf. Accessed August 27, 2016.

30. Klatt S, Fromm M, König J. Transporter-mediated drug-drug interactions with oral antidiabetic drugs. Pharmaceutics. 2011;3(4):680-705.

31. Cycloset $^{\circledR}$ (bromocriptine) tablets [prescribing information]. Laval: Valeant Pharmaceuticals International Inc.; 1978.

32. Kim KA, Park JY. Inhibitory effect of glyburide on human cytochrome p450 isoforms in human liver microsomes. Drug Metab Dispos. 2003; 31(9):1090-1092.

33. Drugbank.ca [homepage on the Internet]. Drugbank: gliquidone. Available from: http://www.drugbank.ca/drugs/DB01251. Accessed August 26, 2016.

34. Cholestage ${ }^{\circledR}$ (colesevelam) film-coated tablets [prescribing information]. Naarden: Genzyme Europe B.V.; 2004.

35. Holstein A, Beil W. Oral antidiabetic drug metabolism: pharmacogenomics and drug interactions. Expert Opin Drug Metab Toxicol. 2009; 5(3):225-241.

36. Schroner Z, Javorsky M, Kozarova M, Tkac I. Pharmacogenetics of oral antidiabetic treatment. Bratisl Lek Listy. 2011;112(8):441-446.

37. Ruzilawati AB, Gan SH. CYP3A4 genetic polymorphism influences repaglinide's pharmacokinetics. Pharmacology. 2010;85(6):357-364.

38. Choi YH, Lee U, Lee BK, Lee MG. Pharmacokinetic interaction between itraconazole and metformin in rats: competitive inhibition of metabolism of each drug by each other via hepatic and intestinal CYP3A1/2. Br J Pharmacol. 2010;161(4):815-829.

39. Lee JH, Kang HE, Lee MG. Pharmacokinetic interaction between telithromycin and metformin in diabetes mellitus rats. Xenobiotica. 2010; 40(3):217-224.

40. Woo SL, Xu H, Li H, et al. Metformin ameliorates hepatic steatosis and inflammation without altering adipose phenotype in diet-induced obesity. PLoS One. 2014;9(3):e91111.

41. Patel K, Foretz M, Marion A, et al. The LKB1-salt-inducible kinase pathway functions as a key gluconeogenic suppressor in the liver. Nat Commun 2014;5:4535

42. Cameron AR, Morrison VL, Levin D, et al. Anti-inflammatory effects of metformin irrespective of diabetes status. Circ Res. 2016;119(5):652-665.

43. Rodbard HW, Jelleinger PS, Davidson JA, et al. Statement by an American Association of Clinical Endocrinologists/American College of Endocrinology Consensus Panel on type 2 diabetes mellitus: an algorithm for glycemic control. Endocr Pract. 2009;15(6):540-559.

44. Stentz FB, Umpierrez GE, Cuervo R, Kitabchi AE. Proinflammatory cytokines, markers of cardiovascular risks, oxidative stress, and lipid peroxidation in patients with hyperglycemic crises. Diabetes. 2004;53(8):2079-2086.

45. Niazi AK, Kalra S. Diabetes and tuberculosis: a review of the role of optimal glycemic control. J Diabetes Metab Disord. 2012;11(1):28.

46. Duckworth WC, Kitabchi AE. Insulin metabolism and degradation Endocr Rev. 1981;2(2):210-233.

47. Ferrannini E, Wahren J, Faber OK, Felig P, Binder C, DeFronzo RA. Splanchnic and renal metabolism of insulin in human subjects: a doseresponse study. Am J Physiol. 1983;244(6):E517-E527.

48. Rabkin R, Ryan MP, Duckworth WC. The renal metabolism of insulin. Diabetologia. 1984;27(3):351-357.

49. Rabkin R, Simon NM, Steiner S, Colwell JA. Effect of renal disease on renal uptake and excretion of insulin in man. N Engl J Med. 1970;282(4): 182-187

50. Humalog ${ }^{\circledR}$ (insulin lispro) injection [prescribing information]. Indianapolis, IN: Eli Lilly and Company; 2011.
51. NovoLog ${ }^{\circledR}$ (insulin aspart) injection [prescribing information]. Princeton, NJ: Novo Nordisk Inc; 2011.

52. Apidra ${ }^{\circledR}$ (insulin glulisine) injection [prescribing information]. Bridgewater, NJ: Sanofi-Aventis; 2009.

53. Helms KL, Kelley KW. Insulin glulisine: an evaluation of its pharmacodynamic properties and clinical application. Ann Pharmacother. 2009;43(4): 658-668.

54. Lantus ${ }^{\circledR}$ (insulin glargine) injection [prescribing information]. Bridgewater, NJ: Sanofi-Aventis; 2007.

55. Levemir ${ }^{\mathbb{B}}$ (insulin detemir) injection [prescribing information]. Bagsvaerd: Novo Nordisk Inc; 2015.

56. Tresiba ${ }^{\circledR}$ (insulin degludec) injection [prescribing information]. Bagsvaerd: Novo Nordisk Inc; 2013.

57. Havelund S, Plum A, Ribel U, et al. The mechanism of protraction of insulin detemir, a long-acting, acylated analog of human insulin. Pharm Res. 2004;21(8):1498-1504.

58. Archer M, Steinvoort C, Oderda G. Glucagon-like peptide-1 (GLP-1) receptor agonists. Drug class review. 2013. Available from: http://www. health.utah.gov/pharmacy/ptcommittee/files/Criteria\%20Review\%20 Documents/05.13/05.13\%20GLP1\%20Receptor\%20Agonist\%20 Drug\%20Class\%20Review.pdf. Accessed August 18, 2016.

59. Saxenda ${ }^{\mathbb{B}}$ (liraglutide) injection [prescribing information]. Bagsvaerd: Novo Nordisk Inc; 2015.

60. SymlinPen ${ }^{\circledR}$ (pramlintide acetate) injection [prescribing information]. London: AstraZeneca; 2015.

61. Fox GJ, Menzies D. A review of the evidence for using bedaquiline (TMC207) to treat multi-drug resistant tuberculosis. Infect Dis Ther. 2013; $2(2): 123-144$.

62. Graham DJ, Staffa JA, Shatin D, et al. Incidence of hospitalized rhabdomyolysis in patients treated with lipid-lowering drugs. JAMA. 2004; 292(21):2585-2090.

63. Colhoun HM, Betteridge DJ, Durrington PN, et al; CARDS Investigators. Primary prevention of cardiovascular disease with atorvastatin in type 2 diabetes in the Collaborative Atorvastatin Diabetes Study (CARDS): multicentre randomised placebo-controlled trial. Lancet. 2004; 364(9435):685-696.

64. Skottheim IB, Gedde-Dahl A, Hejazifar S, Hoel K, Asberg A. Statin induced myotoxicity: the lactone forms are more potent than the acid forms in human skeletal muscle cells in vitro. Eur J Pharm Sci. 2008;33(4-5): 317-325.

65. Marques MP, Coelho EB, Dos Santos NA, Geleilete TJ, Lanchote VL. Dynamic and kinetic disposition of nisoldipine enantiomers in hypertensive patients presenting with type-2 diabetes mellitus. Eur J Clin Pharmacol. 2002;58(9):607-614.

66. Diacon AH, Pym A, Grobusch M, et al. The diarylquinoline TMC207 for multidrug-resistant tuberculosis. $N$ Engl J Med. 2009;360(23): 2397-2405.

67. Diacon AH, Donald PR, Pym A, et al. Randomized pilot trial of eight weeks of bedaquiline (TMC207) treatment for multidrug-resistant tuberculosis: long-term outcome, tolerability, and effect on emergence of drug resistance. Antimicrob Agents Chemother. 2012;56(6):3271-3276.

68. Heller S, Darpö B, Mitchell MI, et al. Considerations for assessing the potential effects of antidiabetes drugs on cardiac ventricular repolarization: a report from the Cardiac Safety Research Consortium. Am Heart J. 2015;170(1):23-35.

69. Segala E, Sougakoff W, Nevejans-Chauffour A, Jarlier V, Petrella S. New mutations in the mycobacterial ATP synthase: new insights into the binding of the diarylquinoline TMC207 to the ATP synthase C-ring structure. Antimicrob Agents Chemother. 2012;56(5):2326-2334.

70. Hartkoorn RC, Uplekar S, Cole ST. Cross-resistance between clofazimine and bedaquiline through upregulation of MmpL5 in Mycobacterium tuberculosis. Antimicrob Agents Chemother. 2014;58(5): 2979-2981.

71. Pym AS, Diacon AH, Tang SJ, et al; TMC207-C209 Study Group. Bedaquiline in the treatment of multidrug- and extensively drug-resistant tuberculosis. Eur Respir J. 2016;47(2):564-574. 
72. Andries K, Villellas C, Coeck N, et al. Acquired resistance of Mycobacterium tuberculosis to bedaquiline. PLoS One. 2014;9(7):e102135.

73. Bloemberg GV, Keller PM, Stucki D, et al. Acquired resistance to bedaquiline and delamanid in therapy for tuberculosis. $N$ Engl J Med. 2015;373(20):1986-1988.

74. Almeida D, Ioerger T, Tyagi S, et al. Mutations in pepQ confer lowlevel resistance to bedaquiline and clofazimine in Mycobacterium tuberculosis. Antimicrob Agents Chemother. 2016;60(8):4590-4599.

75. Diacon AH, Pym A, Grobusch MP, et al; TMC207-C208 Study Group. Multidrug-resistant tuberculosis and culture conversion with bedaquiline. N Engl J Med. 2014;371(8):723-732.

76. Bender $\mathrm{AD}$. The effect of increasing age on the distribution of peripheral blood flow in man. J Am Geriatr Soc. 1965;13(3):192-198.

77. Warren PM, Pepperman MA, Montgomery RD. Age changes in smallintestinal mucosa. Lancet. 1978;2(8094):849-850.

78. Corazza GR, Frazzoni M, Gatto MR, Gasbarrini G. Ageing and smallbowel mucosa: a morphometric study. Gerontology. 1986;32(1): $60-65$.

79. Fülöp T Jr, Wórum I, Csongor J, Fóris G, Leövey A. Body composition in elderly people. I. Determination of body composition by multiisotope method and the elimination kinetics of these isotopes in healthy elderly subjects. Gerontology. 1985;31(1):6-14.

80. Chahine EB, Karaoui LR, Mansour H. Bedaquiline: a novel diarylquinoline for multidrug-resistant tuberculosis. Ann Pharmacother. 2014;48(1): $107-115$.

81. Tolman KG, Fonseca V, Dalpiaz A, Tan MH. Spectrum of liver disease in type 2 diabetes and management of patients with diabetes and liver disease. Diabetes Care. 2007;30(3):734-743.

82. Merrell MD, Cherrington NJ. Drug metabolism alterations in nonalcoholic fatty liver disease. Drug Metab Rev. 2011;43(3):317-334.

83. Rodighiero V. Effects of liver disease on pharmacokinetics. An update. Clin Pharmacokinet. 1999;37(5):399-431.

84. Minaker KL, Rowe JW. Potassium homeostasis during hyperinsulinemia: effect of insulin level, beta-blockade, and age. Am J Physiol. 1982;242(6):E373-E377.

85. Chopra S, Kewal A. Does hypoglycemia cause cardiovascular events? Indian J Endocrinol Metab. 2012;16(1):102-104.

86. Pintavorn P, Cook WJ. Progressive renal insufficiency associated with amiodarone-induced phospholipidosis. Kidney Int. 2008;74(10): $1354-1357$.
87. Leitinger $\mathrm{N}$. The role of phospholipid oxidation products in inflammatory and autoimmune diseases: evidence from animal models and in humans. Subcell Biochem. 2008:49:325-350.

88. Johnson JN, Ackerman MJ. QTc: how long is too long? Br J Sports Med. 2009;43(9):657-662.

89. Iannini PB. Cardiotoxicity of macrolides, ketolides and fluoroquinolones that prolong the QTc interval. Expert Opin Drug Saf. 2002;1(2): $121-128$.

90. Dumaine R, Roy ML, Brown AM. Blockade of HERG and Kv1.5 by ketoconazole. J Pharmacol Exp Ther. 1998;286(2):727-735.

91. Stumpe KO, Kolloch R, Higuchi M, Krück F, Vetter H. Hyperprolactinaemia and antihypertensive effect of bromocriptine in essential hypertension. Identification of abnormal central dopamine control. Lancet. 1977;2(8031):211-214.

92. Sowers JR. Dopaminergic control of circadian norepinephrine levels in patients with essential hypertension. J Clin Endocrinol Metab. 1981; 53(6):1133-1137.

93. Tolman KG, Chandramouli J. Hepatotoxicity of the thiazolidinediones. Clin Liver Dis. 2003;7(2):369-379.

94. Hsiao SH, Liao LH, Cheng PN, Wu TJ. Hepatotoxicity associated with acarbose therapy. Ann Pharmacother. 2006;40(1):151-154.

95. Slim R, Ben Salem C, Zamy M, Biour M. Pioglitazone-induced acute rhabdomyolysis. Diabetes Care. 2009;32(7):e84.

96. $\operatorname{Actos}^{\circledR}$ (pioglitazone) tablets [prescribing information]. Taastrup: Takeda; 2007.

97. Andries K, Verhasselt P, Guillemont J, et al. A diarylquinoline drug active on the ATP synthase of Mycobacterium tuberculosis. Science. 2005;307(5707):223-227.

98. Alsultan A, Peloquin CA. Therapeutic drug monitoring in the treatment of tuberculosis: an update. Drugs. 2014;74(8):839-854.

99. Peloquin CA. Therapeutic drug monitoring in the treatment of tuberculosis. Drugs. 2002;62(15):2169-2183.

100. Heysell SK, Moore JL, Keller SJ, Houpt ER. Therapeutic drug monitoring for slow response to tuberculosis treatment in a state control program, Virginia, USA. Emerg Infect Dis. 2010;16(10):1546-1553.

101. Gualano G, Capone S, Matteelli A, Palmieri F. New antituberculosis drugs: from clinical trial to programmatic use. Infect Dis Rep. 2016; 8(2):6569.
Drug Design, Development and Therapy

\section{Publish your work in this journal}

Drug Design, Development and Therapy is an international, peerreviewed open-access journal that spans the spectrum of drug design and development through to clinical applications. Clinical outcomes, patient safety, and programs for the development and effective, safe, and sustained use of medicines are the features of the journal, which

\section{Dovepress}

has also been accepted for indexing on PubMed Central. The manuscript management system is completely online and includes a very quick and fair peer-review system, which is all easy to use. Visit http://www.dovepress.com/testimonials.php to read real quotes from published authors. 